\title{
On the greatest prime factor of sides of a Heron triangle
}

\author{
Florian Luca \\ Florian Luca received his Ph.D. from the University of Alaska at Fairbanks in 1996. \\ He then held various visiting positions. Since 2000 he works at the Mathematical \\ Institute of the Universidad Nacional Autónoma de México in Morelia. His main fields \\ of research are diophantine equations, and algebraic and combinatorial number theory.
}

\section{Introduction}

A Heron triangle $\triangle$ is a triangle whose sides $a, b, c$ and area

$$
A=\sqrt{s(s-a)(s-b)(s-c)}
$$

are integers. Here, $s=(a+b+c) / 2$ is the semiperimeter of $\triangle$. The triangle is called reduced if $\operatorname{gcd}(a, b, c)=1$. For a positive integer $n$ let $P(n)$ be the largest prime factor of $n$ with the convention that $P(1)=1$. In [5], it was shown that $P(A) \rightarrow \infty$ as $\max \{a, b, c\} \rightarrow \infty$ through triples which are sides of reduced Heron triangles. In [4], it was shown that also $P(a b c) \rightarrow \infty$ again as $\max \{a, b, c\} \rightarrow \infty$ through triples which are sides of reduced Heron triangles. Here, we improve this to:

Theorem 1. $P(b c)$ goes to infinity as $\max \{a, b, c\}$ goes to infinity through triples which are sides of reduced Heron triangles $\triangle$.

The example $a=m^{2}-n^{2}, b=2 m n, c=m^{2}+n^{2}$ with $m=2^{k}$ (with any $k \geq 1$ ) and $n=1$ shows that in the above statement one cannot replace $P(b c)$ by $P(b)$.

Ein Heronsches Dreieck ist ein Dreieck, dessen Seiten und dessen Fläche ganzzahlig sind. Das Heronsche Dreieck wird reduziert genannt, wenn dessen Seitenlängen zueinander teilerfremd sind. Wir sagen, dass ein Dreieck $\triangle$ mit den Seiten $a, b, c$ gegen unendlich strebt, wenn $\max \{a, b, c\} \rightarrow \infty$ gilt. Es ist nun bekannt, dass der grösste Primfaktor des Produkts $a b c$ der Seiten von $\triangle$ gegen unendlich strebt, wenn das Dreieck $\triangle$ eine Folge reduzierter Heronscher Dreiecke durchläuft, die gegen unendlich streben. In der vorliegenden Arbeit verallgemeinert der Autor dieses Ergebnis dahingehend, dass unter den genannten Voraussetzungen auch der grösste Primfaktor des Produkts zweier Seiten von $\triangle$ gegen unendlich strebt. Es ist bekannt, dass ein entsprechendes Resultat für eine einzige Seite von $\triangle$ nicht unbedingt gelten muss. 


\section{Preliminary results}

We shall use some classical results from the theory of Diophantine equations with $\mathcal{S}$-units. Let us first define the concept of $\mathcal{S}$-unit.

Let $\mathbb{K}$ be an algebraic number field. Let $\mathcal{S} \subset \mathbb{K}^{*}$ be a finitely generated subgroup of $\mathbb{K}^{*}$. The elements in $\mathcal{S}$ will be called $\mathcal{S}$-units. Let $s \geq 2$ and $a_{1}, \ldots, a_{s} \in \mathbb{K}^{*}$ be non-zero. The equation

$$
a_{1} x_{1}+\ldots+a_{s} x_{s}=1,
$$

where $x_{1}, \ldots, x_{s} \in \mathcal{S}$ is called an $\mathcal{S}$-unit equation. If $\sum_{i \in I} a_{i} x_{i} \neq 0$ for all non-empty subsets $I \subset\{1, \ldots, s\}$, then the $\mathcal{S}$-unit equation (1) is called non-degenerate.

The most important result about $\mathcal{S}$-unit equations is the following finiteness theorem (see, for example, [3]).

Theorem 2. The non-degenerate $\mathcal{S}$-unit equation (1) has only finitely many solutions $\mathbf{x}=$ $\left(x_{1}, \ldots, x_{s}\right) \in \mathcal{S}^{s}$.

Another result which will be useful to us is the following:

Theorem 3. If $x \in \mathbb{K}, y \in \mathcal{S}$ and $z \in \mathcal{S}$ satisfy

$$
x^{2}=y-z,
$$

then $y / z$ can take only finitely many values.

Proof. Since $\mathcal{S}$ is finitely generated as a group, it follows that there exists a finite set $\mathcal{C} \subset \mathcal{S}$ such that $y=a y_{1}^{5}$ and $z=b z_{1}^{5}$, where $a, b \in \mathcal{C}$ and $y_{1}, z_{1} \in \mathcal{S}$. For example, if $\mathcal{S}$ is generated by $\rho_{1}, \ldots, \rho_{r}$, we can take $\mathcal{C}$ to be the set consisting of $\prod_{i=1}^{r} \rho_{i}^{e_{i}}$, where $e_{i} \in\{0,1, \ldots, 4\}$ for all $i=1, \ldots, r$. Thus, we get the finite collection of Diophantine equations

$$
x^{2}=a y_{1}^{5}-b z_{1}^{5} \quad \text { for } \quad(a, b) \in \mathcal{C}^{2} .
$$

By a theorem of Darmon and Granville [2], each one of the above equations has the property that $y_{1} / z_{1}$ belongs to a finite set. Since $a / b$ can take only finitely many values also, it follows that $y / z=(a / b)\left(y_{1} / z_{1}\right)^{5}$ can take only finitely many values as well.

\section{The proof of Theorem 1}

Assume that $P(b c)$ does not tend to infinity. Let $P \geq 2$ be a number such that $P(b c) \leq P$ holds for infinitely many reduced Heron triangles. Let $\mathbb{K}=\mathbb{Q}[i]$, and let $\mathcal{S}$ be the subgroup of $\mathbb{K}$ generated by the units of $\mathbb{K}$ (which are $\pm 1, \pm i$ ), and by all the primes in $\mathbb{Z}[i]$ of absolute value $\leq P$. Recall that if $p \equiv 3(\bmod 4)$ is a prime number, then $p$ is a prime in $\mathbb{Z}[i]$ also. If $p \equiv 1(\bmod 4)$, then $p=a^{2}+b^{2}=(a+b i)(a-b i)$ and both numbers $a+b i$ and $a-b i$ are primes in $\mathbb{Z}[i]$. Furthermore, $2=i(1-i)^{2}$ and $1-i$ is a prime. Finally, all primes in $\mathbb{Z}[i]$ are obtained as above up to multiplication by units.

Let $(a, b, c)$ be the sides of one of the infinitely many reduced Heron triangles such that $P(b c) \leq P$. Brahmagupta taught us that up to symmetries all reduced Heron triangles $A B C$ have sides of shape

$$
a=(v+w)\left(v w-u^{2}\right), \quad b=v\left(u^{2}+w^{2}\right), \quad c=w\left(u^{2}+v^{2}\right),
$$


where $u, v, w$ are positive integers, $\operatorname{gcd}(u, v, w)=1, v w>u^{2}>v^{2} w /(2 v+w)$ and $v \geq w \geq 1$ (see [1]). Since the above parametrization (2) is given up to permutations of the sides $a, b, c$, we have to consider three different cases.

Case 1. $P(b c) \leq P$.

In this case, $b=v(u+i w)(u-i w) \in \mathcal{S}$, and $c=w(u+i v)(u-i v) \in \mathcal{S}$. It then follows that there exist two numbers $s_{1}$ and $s_{2} \in \mathcal{S}$ such that

$$
u+i w=s_{1}, \quad \text { and } \quad u+i v=s_{2} .
$$

Conjugating the above relations, and eliminating $w$ and $v$, we get

$$
2 u=s_{1}+\bar{s}_{1}=s_{2}+\bar{s}_{2} .
$$

Thus,

$$
s_{1}+\bar{s}_{1}-s_{2}-\bar{s}_{2}=0 .
$$

The above equation is an $\mathcal{S}$-unit equation. We can rewrite it as

$$
s_{1} s_{2}^{-1}+\bar{s}_{1} s_{2}^{-1}-\bar{s}_{2} s_{2}^{-1}=1 .
$$

Assume that equation (3) is non-degenerate. Then, by Theorem 2 , each of $s_{1} / s_{2}, \bar{s}_{1} / s_{2}$ and $\bar{s}_{2} / s_{2}$ can take only finitely many values. In particular, each of $s_{1} / \bar{s}_{1}$ and $s_{2} / \bar{s}_{2}$ can take only finitely many values. Since

$$
\frac{s_{1}}{\bar{s}_{1}}=\frac{u+i w}{u-i w}=\frac{u / w+i}{u / w-i},
$$

it follows that $u / w$ can take only finitely many values. Similarly,

$$
\frac{s_{2}}{\bar{s}_{2}}=\frac{u / v+i}{u / v-i}
$$

so $u / v$ can take only finitely many values. In particular, $v / w=(u / w) /(u / v)$ can take only finitely many values. Now observe that if we scale all three sides $a, b, c$ by $v w^{2}$ we get that the triangle of sides $(a, b, c)$ is similar to the triangle of sides

$$
\left.((v / w)+1)(1-(u / v)(u / w)),(u / w)^{2}+1,(u / v)(u / w)+(v / w)\right),
$$

so our triangle is similar to some triangle from a finite list. Since the triangle is reduced, we get that there are only finitely many possibilities for this triangle.

Thus, it must be the case that equation (3) is degenerate for infinitely many of our triangles. In particular, either $s_{1}=s_{2}$, or $s_{1}=\bar{s}_{2}$, or $s_{2}=\bar{s}_{2}$. The last case is impossible since it leads to $v=0$. The first two cases lead to $\left|s_{1}\right|^{2}=\left|s_{2}\right|^{2}$, therefore $u^{2}+v^{2}=u^{2}+w^{2}$. Hence, $v=w$. Now

$$
u^{2}=\left(u^{2}+v^{2}\right)-v^{2}
$$

and both $y=u^{2}+v^{2}$ and $v^{2}$ are $\mathcal{S}$-units. By Theorem 3, it follows that $\left(u^{2}+v^{2}\right) / v^{2}$ belongs to a finite list. Thus, $u / v$ can take only finitely many values, and since $v=w$, an argument similar to the one above shows that our triangle is similar to one from a finite list. 
Case 2. $P(a b) \leq P$.

Here, we have $v+w \in \mathcal{S}, v \in \mathcal{S}$ and $u^{2}+w^{2}=(u+i w)(u-i w) \in \mathcal{S}$. From the first two relations, we get that $w=s_{1}-s_{2}$, where $s_{1}=v, s_{2}=v+w$ are in $\mathcal{S}$. From the third, we get that $u+i w=s_{3} \in \mathcal{S}$. Thus, $w=\left(s_{3}-\bar{s}_{3}\right) /(2 i)$. Thus, we get the equation

$$
s_{1}-s_{2}=(2 i)^{-1} s_{3}-(2 i)^{-1} \bar{s}_{3} .
$$

Let us show that this $\mathcal{S}$-unit equation is non-degenerate. Indeed, if it were degenerate, then either $s_{1}=s_{2}$, or $s_{1}=(2 i)^{-1} s_{3}$, or $s_{1}=-(2 i)^{-1} \bar{s}_{3}$. The first possibility leads to $s_{3}=\bar{s}_{3}$, therefore $w=0$, which is false. The other two imply that the real part of $s_{3}$ (which is $u$ ) is zero, which is again impossible. Thus, equation (4) is non-degenerate. Rewriting it as

$$
(2 i) s_{1} s_{3}^{-1}-(2 i) s_{2} s_{3}^{-1}+\bar{s}_{3} s_{3}^{-1}=1,
$$

and applying Theorem 2, we get that $s_{1} / s_{3}, s_{2} / s_{3}$ and $\bar{s}_{3} / s_{3}$ belong to a finite list of numbers. Since $\bar{s}_{3} / s_{3}=((u / w)-i) /((u / w)+i)$ belongs to a finite list of numbers, it follows that $u / w$ can take only finitely many values. Furthermore, $1+w / v=s_{2} / s_{1}$ belongs also to a finite list of numbers, therefore $w / v$ can take only finitely many values also. We now get, as in Case 1, that there only finitely many reduced Heron triangles with this property.

Case 3. $P(a c) \leq P$.

This is identical to Case 2 after interchanging $v$ and $w$.

The theorem is therefore proved.

Acknowledgements. This paper was written during Spring 2007 when I visited the Williams College. I thank the Mathematics Department there for its hospitality during my visit.

\section{References}

[1] Carmichael, R.D.: The Theory of Numbers and Diophantine Analysis. New York, Dover 1952.

[2] Darmon, H.; Granville, A.: On the equations $z^{m}=F(x, y)$ and $A x^{p}+B y^{q}=C z^{r}$. Bull. London Math. Soc. 27 (1995), 515-543.

[3] Evertse, J.-H.: The number of solutions of decomposable form equations. Invent. Math. 122 (1995), 559_ 601.

[4] Gaál, I.; Járási, I.; Luca, F.: A remark on prime divisors of lengths of sides of Heron triangles. Experiment. Math. 12 (2003), 303-310.

[5] Kramer, A.-V.; Luca, F.: Some remarks on Heron triangles. Acta Acad. Paedagog. Agriensis Sect. Mat. (N.S.) 27 (2000), 25-38.

Florian Luca

Instituto de Matemáticas

Universidad Nacional Autónoma de México

C.P. 58089

Morelia, Michoacán, México

e-mail: fluca@matmor.unam.mx 